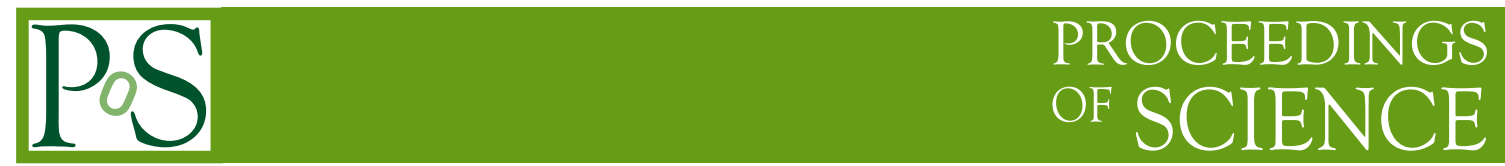

\title{
Minimal Flavour Seesaw Models
}

\section{Daniel Hernandez ${ }^{* \dagger}$}

Theoretical Physics Department, Universidad Autónoma de Madrid

E-mail: d.hernandez@uam.es

\begin{abstract}
We explore realizations of minimal flavour violation (MFV) for the lepton sector. We find that it can be realized within those seesaw models where a separation of the lepton number and lepton flavour violating scales can be achieved. We present a simple implementation of the MFV hypothesis that allows to reconstruct the flavour structure of the model from the values of the light neutrino masses and mixing parameters, even in the presence of $\mathrm{CP}$-violating phases.
\end{abstract}

European Physical Society Europhysics Conference on High Energy Physics, EPS-HEP 2009, July 16 - 222009

Krakow, Poland

\footnotetext{
* Speaker.

†Work in collaboration with M.B. Gavela, T. Hambye, P. Hernandez [1]
} 


\section{Neutrino masses and Minimal Flavour Violation}

Neutrino masses constitute the first evidence of physics beyond the Standard Model (SM). An interesting question is to assess whether this new physics can be tested through low-energy observables beyond neutrino oscillations, such as direct searches for the new particles involved, rare decays or precision electroweak measurements. However, these effects are expected to be undetectable if the new physics scale is orders of magnitude above the $\mathrm{TeV}$, as it is generally required for neutrino mass generation.

In the other hand, if in order to perform such tests we want the new physics scale to be not too far beyond the electroweak scale, we need some symmetry principle to ensure the smallness of neutrino masses. The simplest of these symmetries is a $U(1)$ global lepton number $(\mathrm{LN})$ which forbids the appearance of the dimension five $(d=5)$ Weinberg's effective operator, responsible for light neutrino masses. Thus, it is conceivable a scenario in which new flavour physics would emerge at a scale $\Lambda_{F L}$ at which LN is still an approximate symmetry. LN breaking would result from subtler effects at a still higher energy scale $\Lambda_{L N}$. The typical effective Lagrangian of this theory would have the form

$$
\mathscr{L}=\mathscr{L}_{S M}+\frac{\alpha^{d=5}}{\Lambda_{L N}} \mathscr{O}^{d=5}+\sum_{i} \frac{\alpha_{i}^{d=6}}{\Lambda_{F L}^{2}} \mathscr{O}_{i}^{d=6}+\ldots
$$

where the effective scales $\Lambda_{F L}$ and $\Lambda_{L N}$ take care of the suppressions of each type of contribution and $\Lambda_{L N} \gg \Lambda_{F L}$ is required to obtain tiny neutrino masses. In general, all the effective couplings that break LN, such as the $d=5$ operator, are more suppressed than those that preserve it, such as the $d=6$ one. The latter are also responsible for the rare flavour processes hopefully accessible to near-future experiments.

A very general drawback of models leading to Eq.(1.1) is that there is no relation a priori between the flavour structure of the $d=6$ and $d=5$ operator coeficcients. This lack of predictability can be addressed by adding the so called Minimal Flavour Violation hypothesis into the game.

As it is well known, a large global flavour symmetry would be present in the SM were it not for the presence of the Yukawa couplings, $Y_{u}, Y_{d}$ and $Y_{e}$. Minimal Flavour Violation is the assumption that the Yukawas of the SM are in fact the couplings that break the flavour symmetry in the fundamental theory of the universe, and not just the manisfestation of that breaking at low energies. The Yukawa couplings are then spurions and the coefficients of any flavour charged operator in the low energy theory, such as $\alpha^{d=5}$ and $\alpha^{d=6}$, must be formed by a combination of them. This in turn implies that relations must exist between neutrino masses and mixings and rare flavour processes.

\section{MFV in scalar mediated seesaw models}

The type II seesaw in its basic form only adds one scalar triplet of hypercharge $2,\left(\delta^{++}, \delta^{+}, \delta^{0}\right)$, to the SM field content. Writing this triplet as $\left(\frac{1}{\sqrt{2}}\left(\delta_{1}-i \delta_{2}\right), \delta_{3}, \frac{1}{\sqrt{2}}\left(\delta_{1}+i \delta_{2}\right)\right)$, the most general Lagrangian can be written in terms of $\Delta \equiv\left(\delta_{1}, \delta_{2}, \delta_{3}\right)$ :

$$
\begin{aligned}
\mathscr{L}_{\Delta} & =\left(D_{\mu} \Delta\right)^{\dagger}\left(D^{\mu} \Delta\right)+\left(\overline{\widetilde{\ell_{\mathrm{L}}}} Y_{\Delta}(\tau \cdot \Delta) \ell_{\mathrm{L}}+\mu_{\Delta} \widetilde{\phi}^{\dagger}(\tau \cdot \Delta)^{\dagger} \phi+\text { h.c. }\right)-\Delta^{\dagger} M_{\Delta}{ }^{2} \Delta \\
& -\frac{\lambda_{2}}{2}\left(\Delta^{\dagger} \Delta\right)^{2}-\lambda_{3}\left(\phi^{\dagger} \phi\right)\left(\Delta^{\dagger} \Delta\right)-\frac{\lambda_{4}}{2}\left(\Delta^{\dagger} T^{i} \Delta\right)^{2}-\lambda_{5}\left(\Delta^{\dagger} T^{i} \Delta\right) \phi^{\dagger} \tau^{i} \phi,
\end{aligned}
$$


with $\phi \equiv\left(\phi^{+} \phi^{0}\right)^{T}, T_{i}$ being the three-dimensional representation of the $S U(2)$ generators and $\tau_{i}$ the Pauli matrices. The coexistence of $Y_{\Delta}$ and $\mu_{\Delta}$ explicitly breaks lepton number, inducing at low energies the Weinberg operator:

$$
\delta \mathscr{L}^{d=5}=c_{\alpha \beta}^{d=5}\left(\overline{\ell_{L \alpha}^{c}} \tilde{\phi}^{*}\right)\left(\tilde{\phi}^{\dagger} \ell_{L \beta}\right)+\text { h.c. },
$$

with

$$
c_{\alpha \beta}^{d=5}=2 Y_{\Delta \alpha \beta} \frac{\mu_{\Delta}}{M_{\Delta}^{2}}
$$

As for the generated $d=6$ operators, there is only one at tree level which involves leptons

$$
\delta \mathscr{L}^{d=6}=c_{\alpha \beta \gamma \delta}^{d=6}\left(\overline{\ell_{L}} \gamma_{\mu} \ell_{L \delta}\right)\left(\overline{\ell_{L}} \gamma_{\mu} \ell_{L \gamma}\right)
$$

with

$$
c_{\alpha \beta \gamma \delta}^{d=6}=-\frac{1}{M_{\Delta}^{2}} Y_{\Delta \beta}^{\dagger} Y_{\Delta \delta \gamma} .
$$

Notice that the flavour structure of the $d=6$ coefficient in this model goes essentially as the square of that of the $d=5$. This possibility is the minimal MFV identified in [2]. The type II Seesaw is a concrete model whose low-energy effective theory is such that if we know the flavour structure of the $d=5$ coefficient, then the $d=6$ one is completely determined. This is well known.

The flavour breaking scale $\Lambda_{F L}$ is simply the mass of the triplet. The lepton number violating scale $\Lambda_{L N}$ is a bit subtler since it doesn't correspond to the mass of any new particle. The $\Lambda_{L N}$ scale in eq. (1.1) would correspond instead to the combination $\Lambda_{L N} \sim M_{\Delta}^{2} / \mu_{\Delta}$. Furthermore, a small value for $\mu_{\Delta}$ is stable since setting it to zero restores the lepton number symmetry.

Naive Type-II seesaw presents therefore the two following features. First, there is a hierarchy between the operators that break and preserve lepton number. This hierarchy corresponds to that of the scales $\Lambda_{L N} \gg \Lambda_{F L}$. Second, the model is predictable in the sense that the structure of the $d=6$ flavour coefficients is determined by that of the $d=5$ ones.

\section{The simplest MFV type I seesaw model}

In a typical setup of type I seesaw models, two or more singlet right-handed neutrinos are added to the SM field content. The mass eigenvalues of the Majorana mass matrix of the latter are taken to be at very high scale and the Yukawa couplings are considered of order one. These models lead to an effective theory where $\Lambda_{F L} \sim \Lambda_{L N}$, Eq.(1.1), and are therefore incompatible with large $d=6$ coefficients if they are to explain the smallness of neutrino mass.

Nevertheless, a slight modification of type I seesaw with two right-handed neutrinos succeeds as we show below. Consider the mass Lagrangian

$$
\mathscr{L}_{\text {mass }}=-\left(\begin{array}{lll}
\bar{L}_{L}^{\alpha} \bar{N}^{c}{ }_{R} & \bar{N}^{\prime c}{ }_{R}
\end{array}\right)\left(\begin{array}{ccc}
0 & Y_{N}^{T} v & \varepsilon Y_{N}^{\prime T} v \\
Y_{N} v & \mu^{\prime} & \Lambda \\
\varepsilon Y_{N}^{\prime} v & \Lambda & \mu
\end{array}\right)\left(\begin{array}{c}
L_{L}^{c \alpha} \\
N_{R} \\
N_{R}^{\prime}
\end{array}\right)
$$

where $L_{L}^{\alpha}$ stands for the three lepton families and $v$ is the vacuum expectation value of the Higgs (vev). $Y_{N}$ and $Y_{N}^{\prime}$ are three dimensional vectors. Also $\varepsilon$ is a flavour blind constant; $\varepsilon, \mu$ and $\mu^{\prime}$ are "small parameters", that is, the scales in $\mu, \mu^{\prime}$ are much smaller than those in $\Lambda$ and $v$, and $\varepsilon \ll 1$. 
This ensures an approximate $U(1)_{L N}$ symmetry. The fact that the coupling between $N^{\prime}$ and the $L_{L} \mathrm{~S}$ is assumed small changes the phenomenology radically as we will see.

Neglecting terms of second order in $\varepsilon$ and $\mu / \Lambda$ we have that the coefficients for the $d=5,6$ operators in this mode are given by

$$
\begin{aligned}
& c_{\alpha \beta}^{d=5} \equiv \varepsilon\left(Y_{N}^{\prime T} \frac{1}{\Lambda} Y_{N}+Y_{N}^{T} \frac{1}{\Lambda} Y_{N}^{\prime}\right)_{\alpha \beta}-\left(Y_{N}^{T} \frac{1}{\Lambda} \mu \frac{1}{\Lambda} Y_{N}\right)_{\alpha \beta}, \\
& c_{\alpha \beta}^{d=6} \equiv\left(Y_{N}^{\dagger} \frac{1}{\Lambda^{2}} Y_{N}\right)_{\alpha \beta}+\mathscr{O}(\varepsilon) .
\end{aligned}
$$

And with the redefinition $\tilde{Y}_{N}=Y_{N}^{\prime}-\frac{\mu}{2 \varepsilon \Lambda} Y_{N}$, we can write $c_{\alpha \beta}^{d=5}$ as

$$
c_{\alpha \beta}^{d=5}=\varepsilon\left(\frac{\tilde{Y}_{N}^{T} Y_{N}}{\Lambda}+\frac{Y_{N}^{T} \tilde{Y}_{N}}{\Lambda}\right)
$$

The coefficients in Eq.(3.3) and (3.4) are precisely those that appear in a type I seesaw with the mass matrix

$$
M_{v}=\left(\begin{array}{ccc}
0 & Y_{N}^{T} v & \varepsilon \tilde{Y}_{N}^{T} v \\
Y_{N} v & 0 & \Lambda^{T} \\
\varepsilon Y_{N}^{\prime} v & \Lambda & 0
\end{array}\right)
$$

so, up to $d=6$ and $o(\varepsilon, \mu / \Lambda)$ the tree-level phenomenology of the lepton sectors of the two models Eq.(3.1) and (3.5) are equivalent.

In the simpler model, Eq.(3.5), lepton number is broken due to the simultaneous presence of all three types of terms and light neutrino masses are then expected to depend on $Y_{N}, \tilde{Y}_{N}$ and $\Lambda$. The flavour breaking in this model stems from both $Y_{N}, \tilde{Y}_{N}$, and as a consequence there is flavour violation even in the lepton-number conserving $\varepsilon \rightarrow 0$ limit, as $Y_{N}$ remains active: non-trivial leptonic flavour physics can thus affect processes other than neutrino masses.

The structure of the effective Lagrangian in eq. (1.1) is recovered if one identifies $\Lambda_{F L} \rightarrow \Lambda$ and $\Lambda_{L N} \rightarrow \Lambda / \sqrt{\varepsilon}$. The separation of scales is achieved by having a small $\varepsilon$, which is technically natural since $\varepsilon=0$ restores the lepton number symmetry. The $\Lambda_{L N}$ scale does not correspond to any particle mass at this level, while $\Lambda_{F L}$ corresponds to the Dirac heavy right-handed neutrino mass scale, as expected.

As it turns out, the $d=5$ coefficient in this case has also enough information to reconstruct both Yukawa vectors up to a global renormalization, and therefore, also the flavour structure of $c_{\alpha \beta}^{d=6}[1]$. Let

$$
Y_{N}^{T} \equiv y \mathbf{u} \quad \tilde{Y}_{N}^{T} \equiv \tilde{y} \mathbf{v}
$$

where $y$ and $\tilde{y}$ are real numbers $\langle\mathbf{u}, \mathbf{u}\rangle=\langle\mathbf{v}, \mathbf{v}\rangle=1$. Then, after choosing a particular hierarchy, normal or inverted, the Yukawa couplings can be reconstructed as linear combinations of the columns of the PMNS matrix. Taking the normal hierarchy for illustration purposes we obtain

$$
\begin{aligned}
Y_{N i} & =\frac{y}{\sqrt{2}}\left(\sqrt{1+\rho} U_{i 3}^{*}+\sqrt{1-\rho} U_{i 2}^{*}\right), \\
\tilde{Y}_{N_{i}} & =\frac{\tilde{y}}{\sqrt{2}}\left(\sqrt{1+\rho} U_{i 3}^{*}-\sqrt{1-\rho} U_{i 2}^{*}\right) .
\end{aligned}
$$



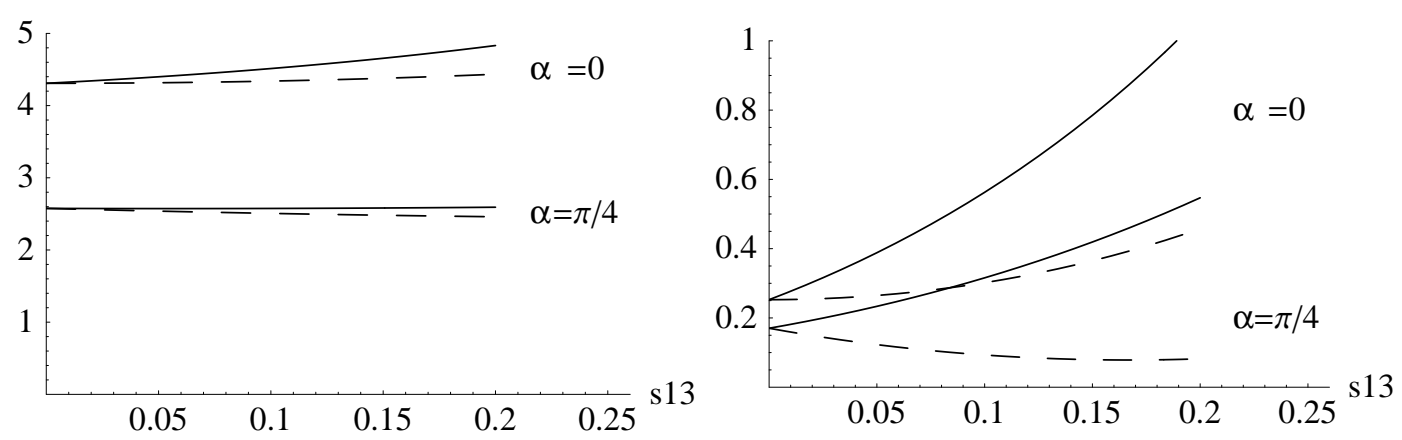

Figure 1: Normal hierarchy. Left: Ratio $B_{e \mu} / B_{e \tau}$ for different values of the CP phase $\delta=0$ (solid) and $\delta=\pi / 2$ (dashed), with $\alpha=0, \pi / 4$ as denoted. Right: the same for the ratio $B_{e \mu} / B_{\mu \tau}$.

where

$$
\rho=\frac{\sqrt{1+r}-\sqrt{r}}{\sqrt{1+r}+\sqrt{r}}, \quad r \equiv \frac{\left|\Delta m_{\text {solar }}^{2}\right|}{\left|\Delta m_{\text {atmos }}^{2}\right|}=\frac{\left|\Delta m_{12}^{2}\right|}{\left|\Delta m_{23}^{2}\right|} .
$$

Furthermore, the masses of the neutrinos are given by

$$
m_{1}=0, \quad\left|m_{2}\right|=\frac{\varepsilon y \tilde{y} v^{2}}{\Lambda}(1-\rho), \quad\left|m_{3}\right|=\frac{\varepsilon y \tilde{y} v^{2}}{\Lambda}(1+\rho),
$$

and using the latter we can fix the combination

$$
\left|\frac{\varepsilon y \tilde{y} v^{2}}{\Lambda}\right| \sim 0.029 \mathrm{eV} \rightarrow\left|\frac{\varepsilon y \tilde{y}}{\Lambda}\right| \sim 4.9 \times 10^{-13} \mathrm{TeV}^{-1} .
$$

From Eq.(3.7) we obtain predictions for the rare decays. We show in Fig. 1 the result for the ratios $B_{e \mu} / B_{e \tau}$ and $B_{e \mu} / B_{\mu \tau}$ as a function of $\theta_{13}$. It is to note the strong dependence on the Majorana phase $\alpha$ as well as on the CP phase for higher values of $\theta_{13}[1]$.

We can briefly review the main results of this work

- The hypothesis of Minimal Flavour Violation is compatible with the standard seesaw model picture of neutrino mass generation. It can be used as a guideline to search for models with high predictability.

- In order to have new flavour physics accesible, another scale apart from the scale of LN violation is required in the fundamental theory: $\Lambda_{F L} \ll \Lambda_{L N}$. Type II seesaw and the type I seesaw examined above are examples of theories in which $\Lambda_{F L}$ is naturally embedded.

- Furthermore, the latter is interesting per se because not only relates $c^{d=5}$ with $c^{d=6}$ but, from the few low-energy observables from neutrino oscillation experiments, it allows as well for the determination of the Yukawas themselves, and therefore of all the flavour physics.

\section{References}

[1] M. B. Gavela, T. Hambye, D. Hernandez and P. Hernandez, JHEP 0909 (2009) 038 [arXiv:0906.1461 [hep-ph]].

[2] V. Cirigliano, B. Grinstein, G. Isidori and M. B. Wise, Nucl. Phys. B 728 (2005) 121 [arXiv:hep-ph/0507001].

[3] E. J. Chun, K. Y. Lee and S. C. Park, Phys. Lett. B 566 (2003) 142 [arXiv:hep-ph/0304069]. 